\title{
An Accelerated Sequential Class to Minimize Combined Risk for Simultaneous Estimation of Parameters of Several Probability Distributions
}

\author{
Rahul Gupta \\ Department of Statistics, University of Jammu \\ Jammu (J\&K)-180006, India \\ rahulgupta68@yahoo.com \\ Yodh Raj \\ Department of Statistics, University of Jammu \\ Jammu (J\&K)-180006, India \\ yodhraj@gmail.com \\ Sunil Kumar \\ Department of Statistics, University of Jammu \\ Jammu (J\&K)-180006, India \\ sunilbhouga106@gmail.com
}

\begin{abstract}
The fixed sample size procedure sometimes fail to deal with the estimation problem in which it is desired to control the combined risk associated with the estimation of parameters of various probability distributions simultaneously. In this paper for a general probabilistic model is proposed an "accelerated" sequential class to minimize the combined risk for simultaneous estimation of parameters of several probability distributions. The proposed class is shown to provide solutions for many estimation problems under different probabilistic setups for "given precision" problems. Besides, positive and negative moments for the stopping times are obtained and they are used for deriving the asymptotic expression for the "regret" associated with the class of "accelerated" sequential estimation procedure.
\end{abstract}

Keywords: Loss function, Accelerated sequential procedure, Asymptotic distribution, Regret.

\subsection{Introduction}

Sometimes, it is desirable to control the combined risk associated with the estimation of parameters of various populations and such problems give rise to the simultaneous estimation problems. The fixed sample size procedures for such problems fail and affirmative solutions are desired. In this direction, Raatikainer(1987) proposed a sequential procedure for simultaneous estimation of percentiles under a general set-up. Then Mukhopadhyay, Hamdy and Darmanto(1988) considered the negative exponential populations and desired the simultaneous confidence interval estimation of the parameters as a follow up of certain selection and ranking problems. Later Mukhopdhyay(1992) provided multi-stage procedures for the simultaneous point estimation of the parameters of several negative exponential populations. For some further work on related simultaneous estimation procedures one can cite the papers of Raatikainer(1993), Mukhopadhyay and Solanky(1998), Aoshima and Mukhopadhyay(1998), Effron(2004) and Ghosh (2005). 
In this paper we have considered a generalized problem of simultaneous estimation of parameters of several populations $f\left(x_{i}, \theta_{i}, \psi_{i}\right), \mathrm{i}=1,2, \ldots \ldots, \mathrm{k}$; where $\theta_{i}$ and $\psi_{i}$ are the unknown parameters, under a family of general loss function and a linear cost function. The problem of fixed sample size procedure to deal with such problem is established in Section 1.2. A class of "accelerated" sequential procedure to tackle the problem is proposed in Section 1.3 and positive and negative moments for the stopping times are obtained and they are used for deriving the asymptotic expression for the "regret" associated with the class of "accelerated" sequential estimation procedure. Finally in Section 1.4.illustrations of estimation problems are provided which can be dealt with the help of the proposed class.

\subsection{The Set-Up of the Problem}

Let $\left(\underline{X}_{i j}\right), j=1,2, \ldots$ be a sequence of independent and identically distributed (iid) random variables from the $i^{\text {th }}, i=1,2,3, \ldots ., k$ t-variate $(\mathrm{t} \geq 1)$ population $\mathrm{f}\left(\underline{\mathrm{X}}_{\mathrm{i}} ; \underline{\theta}_{\mathrm{i}}, \psi_{\mathrm{i}}\right)$, where $\underline{\theta}_{\mathrm{i}}$ and $\psi_{\mathrm{i}}$ are the unknown parameters. Denoting by $\mathrm{R}^{\mathrm{t}}$ and $\mathrm{R}^{+}$, respectively, the $\mathrm{t}$ - dimensional euclidean space and the positive -half of the real line, let $\underline{\theta}_{i} \in \mathrm{R}^{\mathrm{t}}$ and $\psi_{\mathrm{i}} \in \mathrm{R}^{+}$. Having observed a random sample $\underline{X}_{i 1}, \ldots \ldots . ., \underline{X}_{i n_{j}}$ of size $n_{j}(\geq t+1)$ from the $i^{\text {th }}$ population let $\hat{\theta}_{\mathrm{in}_{\mathrm{j}}}=\underline{\hat{\theta}}_{\mathrm{i}}\left(\mathrm{X}_{\mathrm{i} 1}, \ldots \ldots, \mathrm{X}_{\mathrm{in}_{\mathrm{i}}}\right)$ and $\underline{\hat{\psi}}_{\mathrm{in}_{\mathrm{i}}}=\hat{\psi}_{\mathrm{i}}\left(\mathrm{X}_{\mathrm{i} 1}, \ldots, \mathrm{X}_{\mathrm{in}_{\mathrm{i}}}\right)$ be the estimators of $\underline{\theta}_{\mathrm{i}}$ and $\psi_{\mathrm{i}}$, respectively, and satisfying the following assumptions:

$\left(A_{1}\right)$ : There exist a known txt positive definite matrix $Q_{i}$, a number $\delta \in(0,1]$ and an integer

$$
\begin{aligned}
& \mathrm{r}(\geq 1) \text { such that } \\
& \mathrm{n}_{\mathrm{i}} \psi_{\mathrm{i}}^{-1}\left[\left(\underline{\hat{\theta}}_{\mathrm{in}_{\mathrm{i}}}-\underline{\hat{\theta}}_{\mathrm{i}}\right)^{\prime} \mathrm{Q}_{\mathrm{i}}\left(\underline{\hat{\theta}}_{\mathrm{in}_{\mathrm{i}}}-\underline{\hat{\theta}}_{\mathrm{i}}\right)\right]^{\delta} \sim \chi_{\mathrm{r}}^{2},
\end{aligned}
$$

where $\chi_{r}^{2}$ denotes a chi-squarer.v's with $r$ degrees of freedom.

$\left(\mathrm{A}_{2}\right)$ : For all $\mathrm{n}_{\mathrm{i}} \geq \mathrm{m} \geq \mathrm{t}+1, \underline{\hat{\theta}}_{\mathrm{in}_{\mathrm{i}}}$ and $\underline{\hat{\psi}}_{\mathrm{in}_{\mathrm{i}}}$ are stochastically independent.

$\left(A_{3}\right)$ : There exist integers $q(\geq 1)$ and $s(\geq 1)$ such that, for all $n_{i} \geq s+1$,

$$
\mathrm{q}\left(\mathrm{n}_{\mathrm{i}}-\mathrm{s}\right) \underline{\psi}_{\mathrm{in}_{\mathrm{i}}} / \psi_{\mathrm{i}}=\sum_{\mathrm{j}=1}^{\mathrm{n}_{\mathrm{i}}-\mathrm{s}} \mathrm{Z}_{\mathrm{j}}^{\mathrm{q}} \text { with } \mathrm{Z}_{\mathrm{j}}^{\mathrm{q}} \sim \chi_{\mathrm{q}}^{2}
$$

Our goal is to estimate the vector $\underline{\theta}=\left(\underline{\theta}_{1}^{\prime}, \ldots \ldots, \underline{\theta}_{\mathrm{k}}^{\prime}\right)^{\prime}$ pointwise. For $\mathrm{n}=\sum_{\mathrm{i}=1}^{\mathrm{k}} \mathrm{n}_{\mathrm{i}}$, an obvious estimator of $\underline{\theta}$ is $\underline{\hat{\theta}}_{\mathrm{n}}=\left(\underline{\hat{\theta}}_{1 \mathrm{n}_{1}}^{\prime}, \ldots \ldots, \underline{\hat{\theta}}_{\mathrm{kn}_{\mathrm{k}}}^{\prime}\right)^{\prime}$. Let the loss incurred in estimating $\underline{\theta}$ by $\underline{\hat{\theta}}_{\mathrm{n}}$ be

$$
\mathrm{L}\left(\underline{\theta}, \underline{\hat{\theta}}_{\mathrm{n}}\right)=\mathrm{A} \sum_{\mathrm{i}=1}^{\mathrm{k}}\left[\left(\underline{\hat{\theta}}_{\mathrm{in}_{\mathrm{i}}}-\underline{\hat{\theta}}_{\mathrm{i}}\right)^{\prime} \mathrm{Q} \mathrm{Q}_{\mathrm{i}}\left(\underline{\hat{\theta}}_{\mathrm{in}_{\mathrm{i}}}-\underline{\hat{\theta}}_{\mathrm{i}}\right)\right]^{\mathrm{u}}+\mathrm{C}\left(\mathrm{n}_{1}+\ldots . .+\mathrm{n}_{\mathrm{k}}\right),
$$

where $\mathrm{A}(>0)$ is the known weight and $\mathrm{C}(>0)$ is the known cost per unit sample observations from each population.

Utilizing $\left(A_{1}\right)$, the risk corresponding to the loss function (1.1) comes out to be 


$$
\begin{gathered}
R_{n}(c)=K(u, r, \delta) \sum_{i=1}^{k} \psi_{i}^{u} n_{i}^{-u}+C \sum_{i=1}^{k} n_{i}, \text { where } \\
K(u, r, \delta)=2^{u / \delta} A \Gamma(r / 2+u / \delta) / \Gamma(r / 2) .
\end{gathered}
$$

The value $n_{i}^{*}$ of $n_{i}$ from the $i^{\text {th }}$ population, which minimizes the risk (1.2), is given by

$$
n_{i}^{*}=\left\{\frac{u k(u, r, \delta)}{C}\right\}^{1 /(u+1)} \psi_{i}^{u /(u+1)}
$$

And substituting $\mathrm{n}_{\mathrm{i}}=\mathrm{n}_{\mathrm{i}}^{*}$ in (1.2), for $\mathrm{n}^{*}=\sum_{\mathrm{i}=1}^{\mathrm{k}} \mathrm{n}_{\mathrm{i}}^{*}$, the corresponding minimum risk is

$$
R_{n^{*}}(C)=C(1 / u+1) n^{*}
$$

However, in the absence of any knowledge about $\psi_{\mathrm{i}}$ 's, no fixed sample size procedure uniformly minimizes the risk simultaneously for all values of $\psi_{\mathrm{i}}$ 's. In such a situation, motivated by (1.3), we propose the following class of 'accelerated' sequential procedure, determining the sample size as a random variable.

\subsection{The Class C of 'Accelerated' Sequential Procedure}

Take $\mathrm{m} \geq \max \{\mathrm{s}+1, \mathrm{t}+1\}$ to be the initial sample size from each population, where, as in Hall(1983), $\mathrm{m}$ is chosen so as to satisfy $m=o\left(C^{-1 /(u+1)}\right)$ as $C \rightarrow 0$ and $\limsup _{\mathrm{c} \rightarrow 0}\left(\mathrm{~m} / \mathrm{n}_{\mathrm{i}}^{*}\right)<1$. Let $\eta \in(0,1)$ be specified. Start sampling sequentially from the $\mathrm{i}^{\text {th }}$ population, with the stopping time $\mathrm{M}_{\mathrm{i}}$ defined by

$$
\mathrm{M}_{\mathrm{i}}=\inf \left[n_{i} \geq m: n_{i} \geq \eta\left\{\frac{u k(u, r, \delta)}{C}\right\}^{1 /(u+1)} \hat{\psi}_{i\left(n_{i}\right)}^{u /(u+1)}\right]
$$

Based on these $M_{i}$ observations, we compute $\hat{\psi}_{i\left(M_{i}\right)}$. Then we jump ahead and collect $\mathrm{N}_{\mathrm{i}}-\mathrm{M}_{\mathrm{i}}$ more observations from the $\mathrm{i}^{\text {th }}$ population, where

$$
\mathrm{M}_{\mathrm{i}}=\max \left\{M_{i},\left[\left\{\frac{u k(u, r, \delta)}{C}\right\}^{1 /(u+1)} \hat{\psi}_{i\left(n_{i}\right)}^{u /(u+1)}\right]^{+}+1\right\}
$$

After stopping, estimate $\underline{\theta}$ by $\underline{\hat{\theta}}_{(N)}$ where $\mathrm{N}=\sum_{\mathrm{i}=1}^{\mathrm{k}} \mathrm{N}_{\mathrm{i}}$ and $\underline{\hat{\theta}}_{(N)}=\left(\underline{\hat{\theta}}_{\left(1 N_{1}\right)} \ldots \ldots . \hat{\theta}_{k\left(N_{k}\right)}\right)^{\prime}$. 
It can be seen that the risk associated with the class $\mathbf{C}$ of 'accelerated' sequential estimation procedures (1.5)-(1.6) is

$$
R_{N}(c)=c / u \sum_{i=1}^{k} n_{i}^{*(u+1)} E\left(N_{i}^{-u}\right)+c \sum_{i=1}^{k} E\left(N_{i}\right)-c(u+1) n^{*}
$$

with $\mathrm{N}_{\mathrm{i}}$ determined by present rule.

The "Regret" associated with the estimator $\underline{\hat{\theta}}_{(N)}$ of $\underline{\theta}$ is

$$
R_{g}(c)=R_{N}(c)-R_{n^{*}}(c)
$$

Now we state and prove some lemmas.

Lemma 1: For the class Cof accelerated sequential procedures and all

$$
\begin{aligned}
& \mathrm{m}>\max \{\mathrm{t}, \mathrm{s}+2 \mathrm{u} /(\mathrm{u}+1)\}, \text { as } C \rightarrow 0, \\
& \mathrm{E}\left(\mathrm{N}_{\mathrm{i}}\right)=\mathrm{n}_{\mathrm{i}}^{*}\left[\mathrm{~s}+\frac{\mathrm{u}}{\mathrm{q}(\mathrm{u}+1)}\{1+\mathrm{u} /(\mathrm{u}+1)\}\right]+1 / 2+\mathrm{o}(1)
\end{aligned}
$$

and

$$
\operatorname{Var}\left(N_{i}\right)=\frac{2 u^{2}}{\eta q(u+1)^{2}} n_{i}^{*} o\left(C^{-1 /(u+1)}\right)
$$

Proof : Denoting by

$$
U_{M_{i}}=1-\left\{\left\{\frac{u K(u, r, \delta)}{C}\right\}^{1 /(u+1)} \hat{\Psi}_{M_{i}}^{u /(u+1)}-\left[\left\{\frac{u K(u, r, \delta)}{C}\right\}^{1 /(u+1)} \hat{\Psi}_{M_{i}}^{u /(u+1)}\right]^{+}\right\}
$$

We can write

$$
\mathrm{E}\left(\mathrm{N}_{\mathrm{i}}\right)=\mathrm{I}+\mathrm{II}
$$

where

$$
\begin{aligned}
& \mathrm{I}=E\left[N_{i} I\left\{M_{i}>\left[\left[\left\{\frac{u K(u, r, \delta)}{C}\right\}^{1 /(u+1)} \hat{\Psi}_{M_{i}}^{u /(u+1)}\right]^{+}+1\right]\right\}\right] \\
& \mathrm{II}=\left\{\frac{u K(u, r, \delta)}{C}\right\}^{1 /(u+1)} E\left[\hat{\Psi}_{M_{i}}^{u /(u+1)}\right]+E\left(U_{M_{i}}\right)
\end{aligned}
$$

It follows from Hall (1983) that, as $C \rightarrow 0, \mathrm{I}=\mathrm{o}(1)$ and $\mathrm{U}_{\mathrm{M}_{\mathrm{i}}}$ is uniform over $(0,1)$.

Thus, we conclude from (1.11), (1.12) and (1.13) that, as $C \rightarrow 0$,

$$
\mathrm{E}\left(\mathrm{N}_{\mathrm{i}}\right)=\left\{\frac{u K(u, r, \delta)}{C}\right\}^{1 /(u+1)} E\left[\hat{\Psi}_{M_{i}}^{u /(u+1)}\right]+1 / 2+\mathrm{o}(1)
$$


Now we evaluate $\mathrm{E}\left[\widehat{\Psi}_{\mathrm{M}_{\mathrm{i}}}^{\mathrm{u} /(\mathrm{u}+1)}\right]$. To this end, we rewrite the stopping rule (1.5) as

$$
\mathrm{M}_{\mathrm{i}}=\inf \left[\mathrm{n}_{\mathrm{i}} \geq \mathrm{m}-\mathrm{s}: \sum_{\mathrm{j}=1}^{\mathrm{n}_{\mathrm{i}}}\left(\mathrm{q}^{-1} \mathrm{Z}_{\mathrm{j}}^{(\mathrm{q})}\right) \leq\left(\eta \mathrm{n}_{\mathrm{i}}^{*}\right)^{-(\mathrm{u}+) / 1} \mathrm{n}_{\mathrm{i}}^{1+(\mathrm{u}+1) / \mathrm{u}}\left(1+\mathrm{sn}_{\mathrm{i}}^{-1}\right)^{(\mathrm{u}+1) / \mathrm{u}}\right]
$$

Comparing (1.14) with equation (1.1) ofWoodroofe (1977), we obtain in his notations,

$\mathrm{S}_{\mathrm{n}_{\mathrm{i}}}=\sum_{\mathrm{j}=1}^{\mathrm{n}_{\mathrm{i}}}\left(\mathrm{q}^{-1} \mathrm{Z}_{\mathrm{j}}^{(\mathrm{q})}\right), \alpha=1+(\mathrm{u}+1) / \mathrm{u}, \beta=\mathrm{u} /(\mathrm{u}+1), \lambda=\eta \mathrm{n}_{\mathrm{i}}^{*}$

$L\left(n_{i}\right)=\left(1+s n_{i}^{-1}\right)^{(u+1) / u}, \mathrm{~L}_{\mathrm{o}}=\mathrm{s}(\mathrm{u}+1) / \mathrm{u}, \mu=1$, and $\tau^{2}=2 \mathrm{q}^{-1}$. It follows from his Theorem 2.4 that, for all $\mathrm{m}>\{\mathrm{s}+2 \mathrm{u} / \mathrm{q}(\mathrm{u}+1)\}$, as $C \rightarrow 0$,

$$
\mathrm{E}\left(\mathrm{M}_{\mathrm{i}}\right)=\eta \mathrm{n}_{\mathrm{i}}^{*}+\frac{\mathrm{uv}}{(\mathrm{u}+1)}-\mathrm{s}-\frac{\mathrm{u}}{\mathrm{q}(\mathrm{u}+1)}(1+\mathrm{u} /(\mathrm{u}+1))+\mathrm{o}(1),
$$

where $U$ is specified.

Let us consider the difference

$$
D_{c}=M_{i}-\eta\left\{\frac{u K(u, r, \delta)}{C}\right\}^{1 /(u+1)} \hat{\Psi}_{i\left(M_{i}\right)}^{u /(u+1)}
$$

It follows from Woodroofe (1977) that the mean of the asymptotic distribution of $\mathrm{D}_{\mathrm{c}}$ is $\frac{u v}{(u+1)}$.

Thus we obtain from (1.15) and (1.16) that, for all

$$
\begin{aligned}
& m>\{s+2 u / q(u+1)\} \text {, as } C \rightarrow 0, \\
& \left\{\frac{u K(u, r, \delta)}{C}\right\}^{1 /(u+1)} E\left(\hat{\Psi}_{i\left(M_{i}\right)}^{u /(u+1)}\right)=n_{i}^{*}-\eta^{-1}\left[s+\frac{u}{q(u+1)}\{1+u /(u+1)\}\right]+o(1) .
\end{aligned}
$$

Result (1.7) now follows on substituting the value of (1.7) in (1.14).

Let $h\left(M_{i}\right)=\left|M_{i}-\eta n_{i}^{*} /\left(\eta n_{i}^{*}\right)^{1 / 2}\right|$. It follows from a result of Bhattacharya and Mallik (1973) that $h\left(M_{i}\right) \stackrel{L}{\longrightarrow} N\left(0,2 u^{2} / q(u+1)^{2}\right)$, as $c \rightarrow 0$. Moreover, from Theorem1 of Woodroofe(1977), $h^{2}\left(M_{i}\right)$ is uniformly integrable for all $m>\{s+2 u / q(u+1)\}$.

Hence, for all $m>\{s+2 u / q(u+1)\}$, as $\mathrm{C} \rightarrow 0$,

$$
E\left(h^{2}\left(M_{i}\right)\right)=\frac{2 u^{2}}{q(u+1)^{2}}+o(1) \text {. }
$$


And by the definition,

$$
\begin{aligned}
& \operatorname{Var}\left(N_{i}\right)=\eta^{-2} \operatorname{Var}\left(M_{i}\right) . \text { Therefore, } \\
& \operatorname{Var}\left(N_{i}\right)=\eta^{-2}\left[\eta n_{i}^{*}\left\{\frac{2 u^{2}}{\eta q(u+1)^{2}}+o(1)\right\}\right], \text { and (1.9) holds. }
\end{aligned}
$$

Lemma 2: As $C \rightarrow 0, p\left(N_{i} \leq \eta n_{i}^{*}\right)=o\left(C^{q(m-s) / 2 u}\right)$.

Proof: It is easily proved along the lines of Lemma 3 of Chaturvedi, Pandey and Gupta (1991).

Lemma 3: Let the r.v's $\mathrm{W}_{\mathrm{i}}$ 's, $\mathrm{i}=1,2, \ldots, \mathrm{k}$, be defined by $\left|W_{i}-1\right| \leq\left|\left(N_{i} / n_{i}^{*}\right)-1\right|$. Then, for $\delta>0, W_{i}^{\delta-2}$ is uniformly integrable for all $m>\{s+2 u(2-\delta) / q(u+1)\}$.

Proof: On the event $\mathrm{N}_{\mathrm{i}}>\eta \mathrm{n}_{\mathrm{i}}^{*}, W_{i} \leq 2-n$ and $W_{i}^{-1} \leq \eta^{-1}$, so that, on this event, positive, as well as negative, powers of $W_{i}$ are uniformly integrable. Furthermore,on the event $\mathrm{N}_{\mathrm{i}}>\eta \mathrm{n}_{\mathrm{i}}^{*} W_{i} \leq 2$ and $W_{i}^{-1} \leq n_{i}^{*} / m$.

Now, for $\delta<2$, applying Lemma 2, we get from some B $(>0)$,

$$
\begin{aligned}
E\left[W_{i}^{\delta-2} I\left(N_{i} \leq \eta n_{i}^{*}\right)\right] \leq & B n_{i}^{* 2-\delta} p\left(N_{i} \leq \eta n_{i}^{*}\right) \\
& =o\left(c^{q(m-s) / 2 u-(2-\delta) /(u+1)}\right) \\
& =\mathrm{o}(1), \text { for all } \mathrm{m}>\mathrm{s}+2 \mathrm{u}(\mathrm{s}+2 \mathrm{u})(2-\delta) / \mathrm{q}(\mathrm{u}+1) .
\end{aligned}
$$

Finally, for $\delta \geq 2$, once again applying Lemma 2, we get

$$
\begin{aligned}
E\left[W_{i}^{\delta-2} I\left(N_{i} \leq \eta n_{i}^{*}\right)\right] & \leq B p\left(N_{i} \leq \eta n_{i}^{*}\right) \\
& =\mathrm{o}(1), \text { for all } \mathrm{m} \geq \mathrm{s}+1 .
\end{aligned}
$$

And the lemma follows.

Lemma 4: For any $\delta>0$ and all $m>\{s+2 u / q(u+1)\},\{s+2 u(2-\delta) / q(u+1)\}$, as

$$
\begin{aligned}
& C \rightarrow 0, \\
& E\left(N_{i}^{\delta}\right)=n_{i}^{* \delta}+\frac{\delta n_{i}^{* \delta-1}}{\eta q(u+1)^{2}}\left[(\delta-2) u^{2}-(u+1)\{q s(u+1)-u-\eta q(n+1) / 2\}\right] \\
& \quad+o\left(c^{-(\delta-1) /(u+1)}\right)
\end{aligned}
$$

And

$$
\begin{aligned}
E\left(N_{i}^{-\delta}\right)=n_{i}^{*-\delta} & +\frac{\delta n_{i}^{*-(\delta+1)}}{\eta q(u+1)^{2}}\left[(\delta+2) u^{2}-(u+1)\{q s(u+1)+u-\eta q(n+1) / 2\}\right] \\
& +o\left(c^{(\delta+1) /(u+1)}\right)
\end{aligned}
$$


Proof : Using the expression

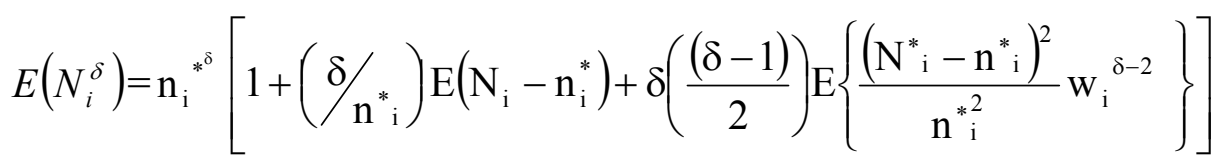

Lemmas 1 and 3 and the fact that $W_{i} \stackrel{\text { a.s }}{\longrightarrow} 1$ as $C \rightarrow 0$, we obtain for all

$$
\begin{gathered}
m>\max \{\{s+2 u / q(u+1)\},\{s+2 u(2-\delta) / q(u+1)\}\}, \text { as } C \rightarrow 0, \\
E\left(N_{i}^{\delta}\right)=n_{i}^{* \delta}+\delta n_{i}^{* \delta-1}\left[-\eta^{-1}\left[s+\frac{u}{q(u+1)}(1+u /(u+1))\right]+1 / 2+o(1)\right] \\
+\frac{\delta(\delta-1)}{2} n_{i}^{* \delta-2}\left[\frac{2 u^{2}}{\eta q(u+1)^{2}} n_{i}^{*}+o\left(c^{-1 /(u+1)}\right)\right] .
\end{gathered}
$$

And (1.19) follows.

Furthermore, using Taylor's expression for $N_{i}^{-\delta}$, Lemma 1 and 3 , and the fact that $W_{i} \stackrel{a . s}{\longrightarrow} 1$ as $C \rightarrow 0$, we obtain for all

$$
\begin{gathered}
m>\max \{\{s+2 u / q(u+1)\},\{s+2 u(2-\delta) / q(u+1)\}\}, \text { as } C \rightarrow 0, \\
E\left(N_{i}^{-\delta}\right)=n_{i}^{*-\delta}+\delta n_{i}^{*-(\delta+1)}\left[-\eta^{-1}\left[s+\frac{u}{q(u+1)}(1+u /(u+1))\right]+1 / 2+o(1)\right] \\
+\frac{\delta(\delta+1)}{2} n_{i}^{*-(\delta+2)}\left[\frac{2 u^{2}}{\eta q(u+1)^{2}} n_{i}^{*}+o\left(c^{-1 /(u+1)}\right)\right],
\end{gathered}
$$

and (1.20) holds.

Now we prove the main theorem of this section, which provides asymptotic expression for the 'regret' corresponding to the class $\mathbf{C}$ of 'accelerated' sequential estimation procedures.

Theorem: For the class $\mathbf{C}$ of 'accelerated' sequential procedures and for all $m>\max \{\{t, s+2 u / q(u+1)\},\{s+2 u(2-\delta) / q(u+1)\}\}$, as $C \rightarrow 0$,

$$
R_{g}(c)=\frac{C k u^{2}}{\eta q(u+1)}+o(C) \text {. }
$$

Proof: Substituting the values of $R_{n}(C)$ and $R_{N}(C)$ from (1.4) and (1.7) in (1.8) respectively, we obtain

$$
R_{g}(C)=(C / u) \sum_{i=1}^{k} n_{i}^{*(u+1)} E\left(N_{i}^{-u}\right)+C \sum_{i=1}^{k} E\left(N_{i}\right)-C(1 / u+1) n^{*} .
$$


Now, applying Lemma 4, we obtain for any $\delta>0$ and all

$$
\begin{aligned}
m>\max & \{\{t, s+2 u / q(u+1)\},\{s+2 u(2-\delta) / q(u+1)\}\}, \text { as } C \rightarrow 0, \text { we get } \\
R_{g}(c)= & (C / u) \sum_{i=1}^{k} n_{i}^{*(u+1)}\left[n_{i}^{*-u}+\frac{u}{\eta q(u+1)^{2}} n_{i}^{*-(u+1)}\left\{(u+2) u^{2}+(u+1)(q s(u+1))+u-\frac{\eta q(u+1)}{2}+o(C)\right\}\right] \\
& +C \sum_{i=1}^{k} n_{i}^{*} \eta^{-1}\left[n_{i}^{*}-\eta^{-1}\left\{s+\frac{u}{q(u+1)^{2}}(1+u /(u+1))\right\}-1 / 2+o(1)\right]-C(1 / u+n) n^{*}
\end{aligned}
$$

and the theorem follows .

\subsection{Estimation Problems Having Solutions Provided by Class C of "Accelerated" Sequential Procedure}

\subsubsection{Simultaneous estimation of the means of several normal populations}

Let us consider a sequence $\left(X_{i j}\right), \mathrm{j}=1,2, \ldots$ of iidr.v's from the $i^{\text {th }}, \mathrm{i}=1,2, \ldots \ldots \mathrm{k}$ univariate normal population $N\left(x_{i}, \mu_{i}, \sigma_{i}^{2}\right)$, where $\mu_{i} \in(-\infty, \infty)$ and $\sigma_{i}^{2} \in(\theta, \infty)$ are the unknown mean and variance, respectively. Having recorded a random sample $X_{i 1}, \ldots \ldots . ., X_{i n_{i}}$ of size $n_{i}(\geq 2)$ from the $i^{\text {th }}$ population, let $\bar{X}_{i\left(n_{i}\right)}=n_{i}^{-1} \sum_{j=1}^{n_{i}} X_{i j}$ and $s_{i\left(n_{i}\right)}^{2}=\left(n_{i}-1\right)^{-1} \sum_{j=1}^{n_{i}}\left(X_{i j}-\bar{X}_{i\left(n_{i}\right)}\right)^{2}$.It can be verified (see Starr(1966)] that $\left(A_{1}\right)-\left(A_{3}\right) \quad$ are $\quad$ satisfied for $\left.\theta_{i}=\mu_{i}, \psi_{i}=\sigma_{i}^{2}, \hat{\hat{\theta}}_{i n_{i}}=\bar{X}_{i\left(n_{i}\right)}, \quad \hat{\Psi}_{i\left(n_{i}\right)}^{2}=s_{i\left(n_{i}\right)}^{2}\right)$ $\mathrm{r}=\mathrm{q}=\mathrm{s}=\mathrm{t}=1, \mathrm{Q}_{\mathrm{i}}=\mathrm{I}_{1 \times 1}=1$ and $\delta=1$.

\subsubsection{Simultaneous estimation of the mean vectors of several multinormal populations}

Let us consider a sequence $\left(\underline{X}_{i j}\right), \mathrm{j}=1,2, \ldots$ of iidr.v's from the $i^{\text {th }}, \mathrm{i}=1,2, \ldots \ldots \mathrm{k}$ univariate normal population $N_{p}\left(x_{i}, \underline{\mu}_{i}, \sigma_{i}^{2} \Sigma_{i}\right)$, where $\underline{\mu}_{i}$ is the px1 unknown mean vector, $\sigma_{i}^{2} \in(0, \infty)$ are the unknown scalar and $\Sigma_{i}$ is a known pxp positive definite matrix. Given a random sample $\underline{X}_{i 1}, \ldots \ldots ., \underline{X}_{i n_{i}}$ of size $n_{i}(\geq p+1)$ from the $i^{\text {th }}$ population, let $\quad \bar{X}_{i\left(n_{i}\right)}=n_{i}^{-1} \sum_{j=1}^{n_{i}} \underline{X}_{i j}$ and $\hat{\sigma}_{i\left(n_{i}\right)}^{2}=\left(p\left(n_{i}-1\right)\right)^{-1} \sum_{j=1}^{n_{i}}\left(\underline{X}_{i j}-\underline{X}_{i\left(n_{i}\right)}\right)^{2}$.It can be verified (see, Wang, 1980) that $\left(A_{1}\right)-\left(A_{3}\right)$ hold for $\theta_{i}=\mu_{i}, \psi_{i}=\sigma_{i}^{2}, \hat{\theta}_{i n_{i}}=$ $\underline{\bar{X}}_{i\left(n_{i}\right)}, \hat{\Psi}_{i\left(n_{i}\right)}^{2}=\hat{\sigma}_{i\left(n_{i}\right)}^{2}, \mathrm{r}=\mathrm{t}=\mathrm{p}, \mathrm{Q}_{\mathrm{i}}=\sum_{i}^{-1}$ and $\delta=1$. 


\subsubsection{Simultaneous estimation of the regression parameters of several linear models}

Consider the $i^{t h}$ linear model $\quad \underline{Y}_{i\left(n_{i}\right)}=\underline{X}_{i\left(n_{i}\right)} \underline{\beta}_{i}+\underline{\Sigma}_{i\left(n_{i}\right)}, \mathrm{i}=1,2, \ldots . . \mathrm{k}$., where $\underline{Y}_{i\left(n_{i}\right)}$ is an observed $n_{i} * 1$ random vector, $\underline{X}_{i\left(n_{i}\right)}$ is a $n_{i} * p$

Matrix of rank $\mathrm{p}, \underline{\beta}_{i}$ is the $p^{*} 1$ vector of unknown regression parameters, and $\underline{\Sigma}_{i\left(n_{i}\right)}$ is the disturbance term following $N_{n_{i}}\left(\underline{0}, \sigma_{i}^{2} I_{n_{i}}\right)$ distribution. The ordinary least squares estimator of $\underline{\beta}_{i}$ is $\underline{\hat{\beta}}_{i\left(n_{i}\right)}=\left(\underline{X}_{i\left(n_{i}\right)}^{\prime} \underline{X}_{i\left(n_{i}\right)}\right)^{-1} \underline{X}_{i\left(n_{i}\right)} \underline{Y}_{i\left(n_{i}\right)} \quad$ and $\quad$ we use $\hat{\sigma}_{i\left(n_{i}\right)}^{2}=\left(n_{i}-p\right)^{-1} \underline{Y}_{i\left(n_{i}\right)}^{\prime}\left[I_{n_{i}}-\underline{X}_{i\left(n_{i}\right)}\left(\underline{X}_{i\left(n_{i}\right)}^{\prime} \underline{X}_{i\left(n_{i}\right)}\right)^{-1} \underline{X}_{i\left(n_{i}\right)}^{\prime} \underline{Y}_{i\left(n_{i}\right)} \quad\right.$ to $\quad$ estimate $\sigma_{i}^{2}$. It can be seen [see, Judge and Bock $\left(1978\right.$, P.20)] that $\left(A_{1}\right)-\left(A_{3}\right)$ are satisfied for $\mathrm{r}=\mathrm{t}=\mathrm{p}, \underline{\theta}_{i}=\underline{\beta}_{i}, \psi_{i}=\sigma_{i}^{2}, \underline{\hat{\theta}}_{i n_{i}}=\underline{\hat{\beta}}_{i\left(n_{i}\right)}$, $\hat{\Psi}_{i\left(n_{i}\right)}^{2}=\hat{\sigma}_{i\left(n_{i}\right)}^{2}, \mathrm{q}=1, \mathrm{~s}=\mathrm{p}, Q_{i}=n_{i}^{-1}\left(\underline{X}_{i\left(n_{i}\right)}^{\prime} \underline{X}_{i\left(n_{i}\right)}\right)^{-1}$ and $\delta=1$.

\subsubsection{Simultaneous estimation of the location parameters of several negative exponential populations}

Let $\left(\underline{X}_{i j}\right), \mathrm{j}=1,2, \ldots$. be a sequence of the iidr.v's from the $i^{\text {th }}$ negative exponential populations,

$f\left(x_{i} ; \underline{\mu}_{i}, \sigma_{i}\right)=\sigma_{i}^{-1} \exp \left[-\left(x_{i}-\mu_{i}\right) / \sigma_{i}\right] ; x_{i} \geq \mu_{i}, \mathrm{i}=1,2, \ldots ., \mathrm{k}, \quad$ where $\mu_{i} \in(-\infty, \infty)$ and $\sigma_{i} \in(0, \infty)$ are the unknown location and scale parameters, respectively. For a random sample $X_{i 1}, \ldots \ldots . ., \quad X_{i n_{i}}$ of size $n_{i}(\geq 2)$ from the $i^{\text {th }}$ population, we define $X_{i\left(n_{i}\right)}^{*}=\min \left(X_{i 1}, \ldots \ldots, X_{i n_{i}}\right)$ and $\hat{\sigma}_{i\left(n_{i}\right)}=\left(n_{i}-1\right)^{-1} \sum_{j=1}^{n_{i}}\left(\underline{X}_{i j}-\underline{X}_{i\left(n_{i}\right)}^{*}\right)$.

Assumptions $\left(A_{1}\right)-\left(A_{3}\right)$ are satisfied [see Basu (1971)] for $\mathrm{r}=2, \mathrm{t}=1, \underline{\theta_{i}}=\mu_{i}, \psi_{i}=\sigma_{i}$, $\underline{\hat{\theta}}_{i n_{i}}=X_{i\left(n_{i}\right)}^{*}, \quad \hat{\Psi}_{i\left(n_{i}\right)}=\sigma_{i\left(n_{i}\right)}^{2}, \mathrm{q}=2, \mathrm{~s}=1, Q_{i}=I_{1 \times 1}=1$ and $\delta=1 / 2$.

\subsubsection{Simultaneous estimation of the parameters of several Pareto distribution}

Let $\left(\underline{X}_{i j}\right), \mathrm{j}=1,2, \ldots$ be a sequence of the iidr.v's from the $i^{\text {th }}(\mathrm{i}=1,2, \ldots ., \mathrm{k})$ first kind of Pareto distribution 
$f\left(x_{i} ; \underline{\mu}_{i}, \sigma_{i}\right)=\sigma_{i}^{-1} \mu_{i}^{-1 / \sigma_{i}} x_{i}^{-1 / \sigma_{i}^{-1}} ; x_{i} \geq \underline{\mu}_{i}>0, \sigma_{i}>0$, where $\underline{\mu}_{i}$ and $\sigma_{i}$ are respectively, unknown scale and shape parameters. Based on random sample $\underline{X}_{i 1}, \ldots \ldots . ., \underline{X}_{i n_{i}}$ of size, for $n_{i}(\geq 2) X_{i\left(n_{i}\right)}^{*}=\min \left(X_{i 1}, \ldots \ldots . ., X_{i n_{i}}\right)$, we use

$$
\mu_{i\left(n_{i}\right)}^{*}=\log X_{i\left(n_{i}\right)}^{*} \text { and } \quad \hat{\sigma}_{i\left(n_{i}\right)}=\left(n_{i}-1\right)^{-1} \sum_{j=1}^{n_{i}} \log X_{i j} / X_{i\left(n_{i}\right)}^{*}
$$

as the estimators of $\log \underline{\mu}_{i}$ and $\sigma_{i}$ are respectively.We observe that [see, Wang (1973)] $\left(A_{1}\right)-\left(A_{3}\right)$ are satisfied for $\mathrm{r}=2, \mathrm{t}=1, \underline{\theta}_{i}=\log \mu_{i}, \psi_{i}=\sigma_{i}, \hat{\theta}_{i n_{i}}=\mu_{i\left(n_{i}\right)}^{*}$, $\hat{\Psi}_{i\left(n_{i}\right)}=\hat{\sigma}_{i\left(n_{i}\right)}, \mathrm{q}=2, \mathrm{~s}=1, Q_{i}=I_{1 \times 1}=1$ and $\delta=1 / 2$.

\subsubsection{Simultaneous estimation of the means of the several inverse Gaussian populations}

Let $\left(\underline{X}_{i j}\right), \mathrm{j}=1,2, \ldots$. be a sequence of the iidr.v's from an inverse Gaussian distribution

$$
f\left(x_{i} ; \underline{\mu}_{i}, \lambda_{i}\right)=\left\{\lambda_{i} / 2 \pi \mathrm{x}_{\mathrm{i}}^{3}\right\}^{1 / 2} \exp \left\{-\frac{\lambda_{i}}{2 \mu_{\mathrm{i}}^{2}} \frac{\left(x_{i}-\mu\right)^{2}}{x_{i}}\right\} ; x_{i}>0,
$$

where $\underline{\mu}_{i} \in(-\infty, \infty)$ and $\lambda_{i}^{-1} \in(0, \infty)$ are the unknown mean and scalar parameters respectively. Given a random sample $\underline{X}_{i 1}, \ldots \ldots . ., \underline{X}_{i n_{i}}$ of size, $n_{i}(\geq 2)$ from the $i^{\text {th }}$ population, we use $\bar{X}_{i\left(n_{i}\right)}=n_{i}^{-1} \sum_{j=1}^{n_{i}} X_{i j}, \hat{\lambda}_{i\left(n_{i}\right)}=\left(n_{i}-1\right)^{-1} \sum_{j=1}^{n_{i}}\left(X_{i j}^{-1}-\bar{X}_{i\left(n_{i}\right)}^{-1}\right)$

as the estimators of $\mu_{i}$ and are $\lambda_{i}^{-1}$ respectively. We observe that (see, Chaturvedi,1985) $\left(A_{1}\right)-\left(A_{3}\right)$ are satisfied for $\mathrm{r}=\mathrm{t}=1, \underline{\theta}_{i}=\mu_{i}, \psi_{i}=\lambda_{i}^{-1}, \underline{\hat{\theta}}_{i n_{i}}=\bar{X}_{i\left(n_{i}\right)}^{*}$, $\hat{\Psi}_{i\left(n_{i}\right)}=\hat{\lambda}_{i\left(n_{i}\right)}, \mathrm{q}=\mathrm{s}=\delta=1$ and $Q_{i\left(n_{i}\right)}=I_{1 \times 1}=1$.

\subsubsection{Simultaneous estimation problem related to multiple comparison procedures}

Let us consider the linear regression models of sections 3. In multiple comparison procedures [see Hochberg and Tamhane(1987)] for a brief discussion], one may be interested in estimating the parametric functions of the components of a $1 \times 1(1 \leq l \leq p)$ subvector $\underline{\beta}_{i}^{*}$ of $\underline{\beta}_{i}$. Let $\underline{\hat{\beta}}_{i\left(n_{i}\right)}^{*}$ be the corresponding subvector of $\underline{\hat{\beta}}_{i\left(n_{i}\right)}$ and $V_{i}$ is 1x1submatrix of $\left(X_{i\left(n_{i}\right)}^{\prime} X_{i\left(n_{i}\right)}\right)^{-1}$ corresponding to the $\underline{\hat{\beta}}_{i\left(n_{i}\right)}^{*}$ part of $\underline{\beta}_{i\left(n_{i}\right)}$. For a known 
p x1 matrix $G_{i}=\left(\underline{g_{i 1}^{\prime}}, \ldots \ldots, \underline{g_{i p}^{\prime}}\right)^{\prime}, \underline{\gamma}_{i}=\left(\underline{g}_{i 1}^{\prime} \underline{\beta}_{i}^{*}, \ldots \ldots, \underline{g}_{i p}^{\prime} \underline{\beta}_{i}^{*}\right)=G_{i} \underline{\beta}_{i}^{*}$. We use the leastsquares estimator $\underline{\gamma}_{i}=G_{i} \underline{\hat{\beta}}_{i\left(n_{i}\right)}^{*}$ to estimated by $s_{i\left(n_{i}\right)}^{2}=v_{i}^{-1}\left\|\underline{Y}_{i}-\underline{\hat{Y}}_{\left(n_{i}\right)}\right\|^{2}$, where $\underline{\hat{Y}}_{i\left(n_{i}\right)}=$ $\underline{X}_{i\left(n_{i}\right)} \underline{\hat{\beta}}_{i\left(n_{i}\right)}, v=n_{i}-s_{i o}$ is the error degree of freedom and $s_{i o}=\operatorname{rank}\left(X_{i\left(n_{i}\right)}\right)$ .Assumptions $\left(A_{1}\right)-\left(A_{3}\right)$ are satisfied for $\mathrm{r}=\mathrm{t}=\mathrm{p}, \underline{\theta}_{i}=\underline{\gamma}_{i}, \psi_{i}=\sigma_{i}^{2}, \underline{\hat{\theta}}_{i n_{i}}=\underline{\hat{Y}}_{i\left(n_{i}\right)}$, $\hat{\Psi}_{i\left(n_{i}\right)}=s_{i\left(n_{i}\right)}^{2}, \mathrm{q}=1, \mathrm{~s}=s_{i o}, \delta=1$ and $Q_{i}=\left(G_{i} V_{i} G_{i}^{\prime}\right)^{-1}$.

\subsubsection{Simultaneous estimation of the means of several random one-way models}

Let us consider the $i^{\text {th }}(\mathrm{i}=1,2, \ldots \ldots, \mathrm{k})$ random one way model:

$$
Y_{i j l}=\mu_{i}+\tau_{i j}+\varepsilon_{i j l}, \mathrm{j}=1,2, \ldots \ldots, n_{i} ; \mathrm{l}=1, \ldots \ldots, r_{i},
$$

where $\tau_{i j}$ 's are independently distributed as $N\left(\theta, \sigma_{i l}^{2}\right), \varepsilon_{i j l}$ 's are independently distributed as $N\left(\theta, \sigma_{i e}^{2}\right), \tau_{i j}$ and $\varepsilon_{i j l}$ are independent for all $\mathrm{i}, \mathrm{j}$ and 1 and $\sigma_{i j} \in(0, \infty)$ and $\sigma_{i e} \in(0, \infty)$ are unknown. We assume that $r_{i}$-the number of samples per treatment is known. Let $\bar{Y}_{i}=\left(n_{i} r_{i}\right)^{-1} \sum_{j=1}^{n_{i}} \sum_{l=1}^{r_{i}} Y_{i j l}$ be the over all sample mean from the $i^{\text {th }}$ population and MST and MSE denotes, respectively, the usual ANOVA mean squares for the treatments and errors. We notice that the assumptions $\left(A_{1}\right)-\left(A_{3}\right)$ are valid for $\mathrm{r}=\mathrm{t}=1$, $\underline{\theta}_{i}=\underline{\mu}_{i}, \psi_{i}=\left(r_{i} \sigma_{i l}^{2}+\sigma_{i e}^{2}\right), \underline{\hat{\theta}}_{i n_{i}}=\underline{\hat{Y}}_{i}$, $\hat{\Psi}_{i\left(n_{i}\right)}=\mathrm{MST}=r_{i} \sum_{j=1}^{n_{i}} \frac{\left(\bar{Y}_{i j .}-\bar{Y}_{i}\right)^{2}}{\left(n_{i}-1\right)}, \mathrm{q}=n_{i}, \mathrm{~s}=\delta=1$ and $Q_{i}=r_{i} I_{1 \times 1}=r_{i}$.

\section{References}

1. Basu, A.P. (1971). On a sequential rule for estimating the location parameter of an exponential distribution. Nav.Res.Logist.Quart., 18,329-337.

2. Bhattacharya, P.K. and Mallik, A. (1973).Asymptotic normality of the stopping times of some sequential procedures. Ann. Statist., 1, 1203-1211.

3. Chaturvedi, A. (1985). Sequential estimation of an inverse Gaussian distribution with prescribed proportional closeness. Cal. Statist. Assoc. Bull., 35, 215-219.

4. Chaturvedi, A. Pandey, S.K. and Gupta, M.(1991).On a class of asymptotically risk-efficient sequential procedures. Scand, Actur. Jour., 1:87-96.

5. Ghosh, D. (2005). Simultaneous estimation procedures and multiple testing: a decision- theoretic framework. Working Paper Series- Working Paper 54. The University of Michigan-Department of Statistics. 
6. Efron, B. (2004). Selection and estimation for large scale simultaneous inference. estimation of the mean. Jour. Amer. Statist. Asso., 96, 96-104.

7. Hall, P. (1983). Sequential estimation saving sampling operations. Jour.Roy.Statist.Soc. B 45, 303-310.

8. Hochberg, Y. and Tamhane, A.C. (1987). Multiple comparison procedures. John Wiley and Sons Inc., U.S.A.

9. Judge, George G. and Bock, M.E. (1978). The Statistical Economics. NorthHillPublishing Company, New York, U.S.A.

10. Raatikainer, K.E.E. (1987). Sequential procedure for simultaneous estimation of several percentiles. Simulation, Vol. 49, 4,159-164.

11. Raatikainer, K.E.E. (1993). A sequential procedure for simultaneous estimation of several means. ACM Trans. On Modelling and Comp. Simulation., 3,2,108-133.

12. Aoshima, M. and Mukhopdhyay, N. (1998). Fixed width simultaneous confidence intervals for multinormal means in several intraclass correlation models. Jour.Multiv.Anal.,66,1, 46-63.

13. Mukhopdhyay, N. (1992). Simultaneous point estimation problems for two parameter negative exponential population. Jour.Ind.Statist.Assoc., 30, 33-41.

14. Mukhopdhyay.N, Hamdy, H.I and Darmanto,S. (1988).Simultaneous estimation after selection ranking and other procedures: the negative exponential case. Metrika, 35, 275-286.

15. Mukhopdhyay, N. and Solanky, T.K.S. (1998). Multi-stage methodologies for fixed with simultaneous confidence intervals for all pairwise comparisons. Jour. Stat. Plan. Infer., 73, 1-2, 163-176.

16. Starr, N. (1966). The performance of a sequential procedure for the fixed width interval estimation of the mean. Ann.Math.Statist., 37, 36-50.

17. Wang, Y.H. (1973).Sequential estimation of the scale parameters of the Paretodistribution. Commun. Statist.-Theo. Math., A2, 145-154.

18. Wang, Y.H. (1980). Sequential estimation of the mean of a multinormal population. Jour. Amer. Statist. Assoc., 75, 977-983.

19. Woodroofe, M. (1977). Second order approximations for sequential point and interval Ann.Statist., 5, 984-995. 